Clinical Research

\title{
Effects of daily consumption of the probiotic Bifidobacterium animalis subsp. lactis CECT 8145 on anthropometric adiposity biomarkers in abdominally obese subjects: a randomized controlled trial
}

\author{
Anna Pedret ${ }^{1,2} \cdot$ Rosa M. Valls ${ }^{2,1} \cdot$ Lorena Calderón-Pérez ${ }^{2,1} \cdot$ Elisabet Llauradó $^{2,1} \cdot$ Judit Companys $^{1,2}$. \\ Laura Pla-Pagà 1,2 Ana Moragas ${ }^{4,5,6} \cdot$ Francisco Martín-Luján 4,5,7 Yolanda Ortega ${ }^{4,5,8}$ - Montse Giralt ${ }^{2,1}$. \\ Antoni Caimari ${ }^{1}$ - Empar Chenoll ${ }^{9}$. Salvador Genovés ${ }^{9} \cdot$ Patricia Martorell $^{9}$ - Francisco M. Codoñer ${ }^{10}$. \\ Daniel Ramón ${ }^{9,1} \cdot$ Lluís Arola ${ }^{1,11} \cdot$ Rosa Solà ${ }^{1,2,3}$
}

Received: 21 May 2018 / Revised: 25 July 2018 / Accepted: 29 August 2018 / Published online: 27 September 2018

(c) The Author(s) 2018. This article is published with open access

\begin{abstract}
Background The effects of probiotic Bifidobacterium animalis subsp. lactis CECT 8145 (Ba8145) and those of its heatkilled form (h-k Ba8145) on human anthropometric adiposity biomarkers are unknown.

Objective To assess the effect of Ba8145 and h-k Ba8145 ingestion on anthropometric adiposity biomarkers.

Design Randomized, parallel, double-blind, placebo-controlled trial with abdominally obese individuals. Participants $(n=135)$ consumed 1 capsule/day containing $10^{10}$ colony forming unit (CFU) of Ba8145, $10^{10} \mathrm{CFU}$ of h-k Ba8145, or placebo (maltodextrin) for 3 months.

Results Ba8145 ingestion decreased waist circumference, waist circumference/height ratio, and Conicity index $(P<0.05)$ versus its baseline. Changes versus the placebo group reached significance $(P<0.05)$ after the $\mathrm{h}-\mathrm{k}$ Ba8145 treatment. Ba8145 decreased the body mass index compared with baseline and placebo group $(P<0.05)$. The decrease in visceral fat area after Ba8145 treatments reached significance $(P<0.05)$ only after $\mathrm{h}-\mathrm{k}$ Ba8145. When analyses by gender were performed, significance remained only for women. Diastolic blood pressure and HOMA index decreased $(P<0.05)$ after $\mathrm{h}-\mathrm{k}$ Ba8145. Gut microbiome analyses showed an increase in Akkermansia spp. after Ba8145 treatment, particularly in the live form, which was inversely related to weight $(P=0.003)$.

Conclusions In abdominally obese individuals, consumption of Ba8145, both as viable and mainly as heat-killed cells, improves anthropometric adiposity biomarkers, particularly in women. An increase in the gut Akkermansia genus appears as a possible mechanism involved. Our results support Ba8145 probiotic as a complementary strategy in obesity management.
\end{abstract}

Sources of support : We received funds from the CDTI agency of the Spanish Ministry of Economy and Competitiveness, under the CIEN project SMARTFOODS.

Electronic supplementary material The online version of this article (https://doi.org/10.1038/s41366-018-0220-0) contains supplementary material, which is available to authorized users.

Rosa M. Valls

rosamaria.valls@urv.cat

Extended author information available on the last page of the article.

\section{Introduction}

The rising prevalence of overweight and obesity in Western countries is considered to be pandemic [1]. Recent guidelines of the American ACCE/ACE point out the relevance of tailored lifestyle interventions, such as physical activity and nutrition to tackle overweight and obesity [2]. Probiotics are recognized functional foods with protective effects against obesity and related cardiometabolic conditions [3]. There is a growing interest in knowing whether the beneficial properties of probiotics are preserved after killing the microorganisms (e.g., by heating or sonication) [4]. The effects of the probiotic Bifidobacterium animalis subsp. lactis CECT 8145 (Ba8145) and those of its 
heat-killed form (h-k Ba8145) on human adiposity biomarkers are unknown. Our preclinical data indicated that both forms of this bacterial strain decreased the body fat content, improved lipid profile, insulin sensitivity, and antioxidant status, and modulated satiety markers related to tryptophan metabolism [5, 6]. Consequently, we have assessed the effects of both Ba8145 and h-k Ba8145 on anthropometric adiposity and cardiovascular risk biomarkers, and on the gut microbiome in individuals with abdominal obesity.

\section{Methods}

The isolation and growth of strain Ba8145 has been described previously [5]. The strain was inactivated by heat treatment $\left(121^{\circ} \mathrm{C}, 30 \mathrm{~min}\right)$. Products were administered in opaque hypromellose capsules as: i) placebo $(300 \mathrm{mg}$ of maltodextrin), ii) $\mathrm{Ba} 8145,100 \mathrm{mg}$ of the live strain, $10^{10}$ colony forming unit (CFU)/capsule containing maltodextrin $200 \mathrm{mg}$, or iii) h-k Ba8145, $100 \mathrm{mg}$ of heat-killed CECT 8145 strain at a concentration of $10^{10} \mathrm{CFU}$ before the heat treatment/capsule containing maltodextrin $200 \mathrm{mg}$.

Abdominally obese subjects were recruited between September 2016 and March 2017. Inclusion criteria were: to be aged $>18$ with a waist circumference (WC) $\geq 102 \mathrm{~cm}$ for men and $\geq 88 \mathrm{~cm}$ for women, according to the European guidelines [7]. Exclusion criteria are defined in Supplementary Item 1. Participants signed informed consent prior to participation. The study was approved by the Clinical Research Ethical Committee of the Hospital Universitari Sant Joan, Reus, Spain. The protocol and trial were in accordance with the Helsinki Declaration and Good Clinical Practice Guidelines of the International Conference of Harmonization (ICH GCP). Trial registration: ClinicalTrials.gov: number. NCT02921217.

A randomized, parallel, double-blind, placebo-controlled clinical trial was conducted with: placebo, Ba8145, and h-k Ba8145. Participants ingested 1 capsule/day with water for 3 months. The randomization allocation sequence was generated with a SAS 9.2 (Cary, NC: 83 SAS Institute Inc.) statistical software PROC PLAN. Blinding was maintained by using opaque capsules that did not differ among placebo and Ba8145 treatments. A 3-day dietary record and a Physical Activity Questionnaire Class AF [8] were administered to the participants at baseline and at the end of the study. Dietary recommendations were provided according to guidelines of the 2013 Adult Treatment Panel (ATP III). A fasting blood sample was obtained at baseline and after 6 and 12 weeks of each intervention. Samples were stored at $-80^{\circ} \mathrm{C}$ in the central laboratory's Biobanc-REUS-IISPV (bancmb@grupsagessa.com) until required for batch analyses.
Abdominal visceral fat area (VFA) and subcutaneous fat area (SFA), as well as anthropometric adiposity biomarkers were measured at the beginning and at the end of the 3month intervention periods. Anthropometric adiposity biomarkers other than VFA and SFA were also measured at a 6-week check-up. VFA and SFA were evaluated by magnetic resonance imaging (MRI), transverse body scan in one axial slice $5 \mathrm{~cm}$ over L5-S1 [9]. Anthropometric data were obtained with participants wearing lightweight clothing and no shoes. Waist circumference (WC) was measured at the umbilicus using a $150-\mathrm{cm}$ anthropometric steel measuring tape. Body mass index (BMI) was calculated as the ratio between measured weight $(\mathrm{kg}) /$ and the square of height $(\mathrm{m})$. The waist $(\mathrm{WC}, \mathrm{cm})$ to height $(\mathrm{cm})$ ratio $(\mathrm{WHtR})$ and the conicity index (CI) (WC (m)/ (0.109x square root of weight $(\mathrm{kg}) /$ height $(\mathrm{m}))$ were calculated [10]. Systolic and diastolic blood pressure (SBP and DBP) were measured twice after 2-5 min of patient respite, seated, with 1-min interval in between, using an automatic sphygmomanometer (OMRON HEM-907; Peroxfarma, Barcelona, Spain). The mean values were used. Serum lipids and apolipoproteins, nonesterified fatty acids, glucose, and insulin concentrations were measured in serum by standardized enzymatic automated methods in an autoanalyzer (Beckman Coulter-Synchron, Galway, Ireland). C-reactive protein was determined by high-sensitivity immunoturbidimetry on an autoanalyzer (Roche Diagnostics Systems, Madrid, Spain). Leptin serum concentrations were determined by Elisa Kit (EMD Millipore Corporation, Billerica, USA). At the beginning and at the end of the study, a stool sample was taken with the Protocult $^{\mathrm{TM}}$ collection device and frozen. DNA from stool samples was isolated, sequenced, and bioinformatically analyzed as previously described [11]. Sample size calculation and statistical analyses are described in Supplementary Item 2 .

\section{Results}

From the 187 subjects assessed for eligibility, finally 126 of them $(93 \%, 43$ men and 83 women) received allocated intervention in either placebo $(n=40), \mathrm{Ba} 8145(n=42)$, or h-k Ba8145 $(n=44)$ groups (see CONSORT Flowchart of the study in Supplementary Fig. 1). No differences in baseline characteristics were observed among intervention groups (Supplementary Item 3). Diet and physical activity were similar among groups, but fiber intake was greater in the h-k Ba8145 group versus the placebo group $(P=0.012)$ (Supplementary Item 4).

Both Ba8145 treatments decreased VFA, but significance was achieved only in the case of h-k Ba8145 $(P<0.05)$ with a borderline significance $(P=0.086)$ versus changes in the placebo group when adjusted for WC (Table 1). No changes 


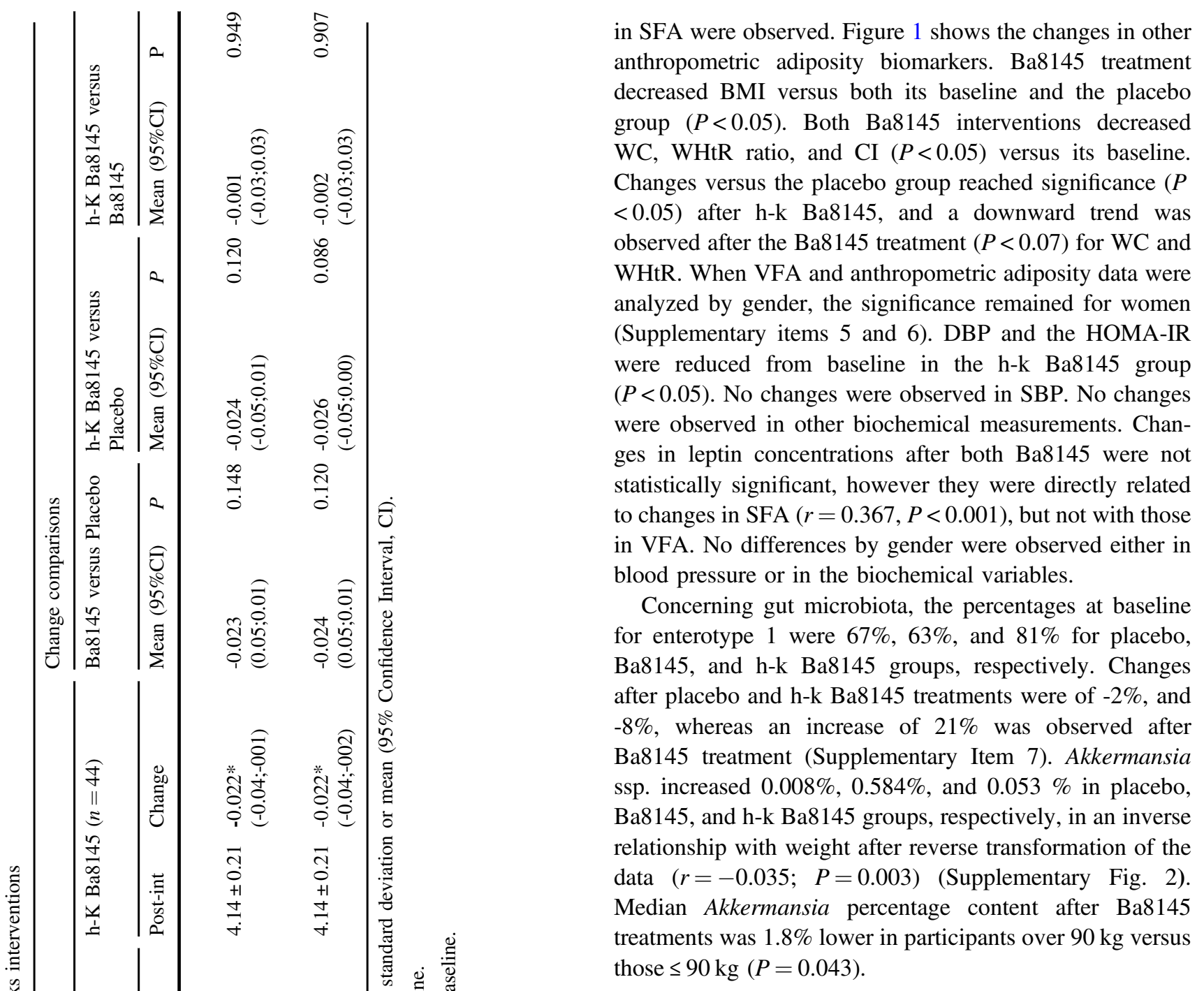

\section{Discussion}

This 3-month intervention study assessed the effect of oral Ba8145 and its h-k Ba8145 form on anthropometric adiposity biomarkers and cardiovascular risk factors. Ba8145 treatments reduced VFA, BMI, WC, WHtR ratio, and CI when compared from baseline to the end of the study, particularly in women. With exception of BMI, the greatest reductions were observed after the h-k Ba8145 intervention. After h-k Ba8145 intervention, DBP and the HOMA index both decreased.

Ba8145 treatment decreased the BMI by $-0.349 \mathrm{~kg} / \mathrm{m}^{2}$. This reduction was similar to those obtained after 3 months of Lactobacillus gasseri SBT2055 $\left(-0.5 \mathrm{~kg} / \mathrm{m}^{2}\right)$ [12], or $L$. plantarum TENSIA $\left(-0.4 \mathrm{~kg} / \mathrm{m}^{2}\right)$ [13] after 3 weeks, and around to those obtained after probiotic consumption in a recent meta-analysis $\left(-0.27 \mathrm{~kg} / \mathrm{m}^{2}\right)$ [6], or after 6-12 months of a multifactorial lifestyle intervention $\left(-0.41 \mathrm{~kg} / \mathrm{m}^{2}\right)$ [14] Average reductions in $\mathrm{WC}$ of $-1.75 \mathrm{~cm}$ for $\mathrm{Ba} 8145$ and 


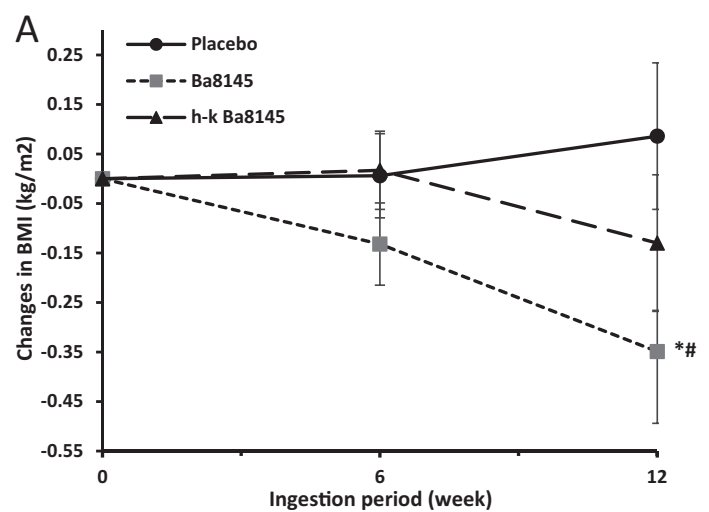

C

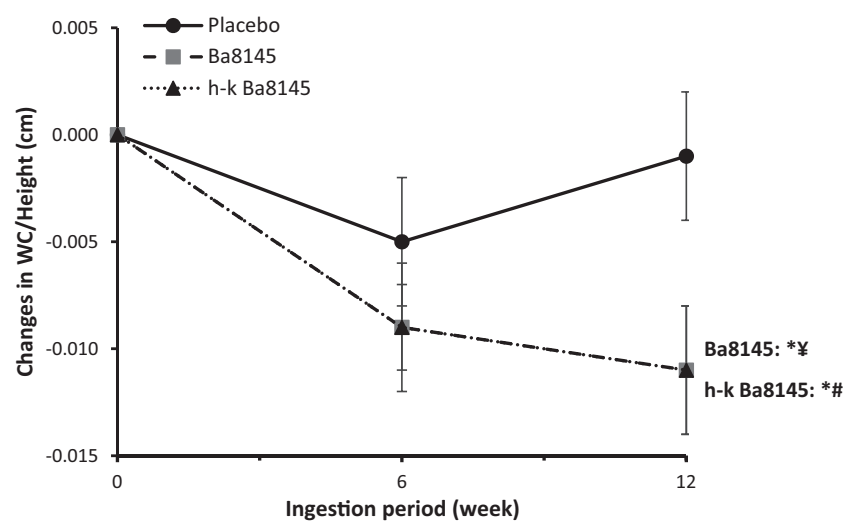

Fig. 1 Changes in anthropometric adiposity biomarkers from baseline to the end of the study. a body mass index, $\mathbf{b}$ waist circumference, c waist circumference/height ratio, $\mathbf{d}$ conicity index. $* P<0.05$ for

$-1.84 \mathrm{~cm}$ for $\mathrm{h}-\mathrm{k} \mathrm{Ba} 8145$ were in the range to those reported after probiotic treatments $[12,15]$ or $6-12$ months of multifactorial lifestyle interventions [14, 16]. The average changes in WHtR (-0.011) and CI (-0.018) were similar to those reported after an 8-week consumption period of a probiotic mix of Lactobacillus and Bifidobacterium (-0.01 and -0.02 for WHtR and CI, respectively) [17]. Mean VFA changes, $-3.66 \mathrm{~cm}^{2}$ after Ba8145 and $-7.01 \mathrm{~cm}^{2}$ for $\mathrm{h}-\mathrm{k}$ $\mathrm{Ba8145}$, were in the range to those reported after Bifidobacterium lactis GCL2505 $\left(-6.6 \mathrm{~cm}^{2}\right)[18]$ during 8 and 12 weeks or Lactobacillus gasseri SBT2055 $\left(-5.6 \mathrm{~cm}^{2}\right)$ [12]. Adiposity accumulation is an independent risk factor for coronary heart disease, hypertension, type-2 diabetes, and impaired glucose tolerance [3]. Accordingly, in the present study, a decrease in DBP and HOMA-IR, a surrogate marker of insulin resistance, was observed after h-k Ba8145 intervention. In our study although no differences were observed between Ba8145 treatments, the heat-killed form appears to be more effective that the live one. The fact that modified probiotics proved more efficient than living strains has been described previously [4]. Mechanisms involved have been related to the host immune system
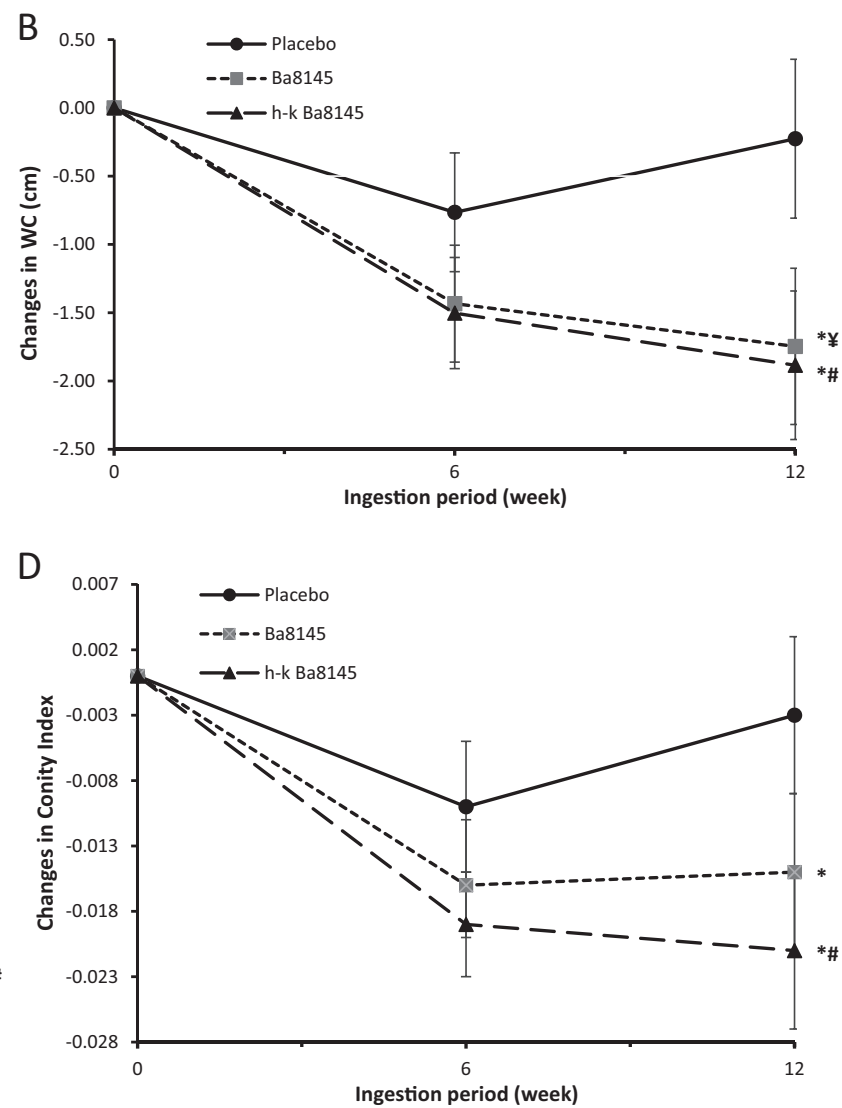

intra-treatment comparisons. \# $P<0.05$ for inter-treatment comparisons (vs. Placebo). $¥ P<0.07$ a trend for inter-treatment comparisons (vs Placebo)

regulation [4, 19]. An increase in leptin sensitivity due to probiotic administration cannot be discarded [20] despite the fact that Ba8145 treatments failed to modify leptin concentrations, possibly due to the lack of changes in SFA, which is the main source for leptin [21].

Previous reports in animal models described a protective effect of Akkermansia on obesity [22]. However, data on the issue from human clinical trials are scarce [23]. In our trial, and concomitantly with the decrease in obesity biomarkers, Ba8145 treatments increased the incidence of Akkermansia spp. in the gut in an inverse relationship with weight decrease. Consistent with this fact, the maximum increase in Akkermansia spp. was observed after the Ba8145 live-form administration, when the maximum decrease in BMI occurred. Also, Akkermansia content was $1.8 \%$ lower in participants over $90 \mathrm{~kg}$ after Ba8145 treatments. The shift to enterotype 1 after Ba8145 treatment could be related with the Bifidobacterium genus, since it promotes growth of Bacteroides, the main player in enterotype 1 .

The study has strengths and limitations (Supplementary Item 8). One limitation is the inability to assess potential interactions between capsules and other dietary components. 
Also, the fact that most of the subjects enrolled in the study were female could account for the lack of significance of changes in the male group. One strength of the study lies in the fact that the probiotic strains were administered alone. In summary, this study shows that ingestion of the probiotic Bifidobacterium animalis subsp. lactis CECT 8145, as viable cells and mainly in a heat-killed form, reduces anthropometric adiposity biomarkers in abdominally obese individuals, particularly in women. An increase in gut Akkermansia genus appears as a possible mechanism involved. Our results support the use of Ba8145 probiotic as a complementary strategy in the management of obesity.

\section{Compliance with ethical standards}

Conflict of interest Empar Chenoll, Salvador Genovés, Patricia Martorell, Francisco M. Codoñer and Daniel Ramón are employees of Biopolis SL/ADM or Lifesequencing/ADM, Valencia, Spain. All the other authors declare that they have no conflict of interest.

Open Access This article is licensed under a Creative Commons Attribution 4.0 International License, which permits use, sharing, adaptation, distribution and reproduction in any medium or format, as long as you give appropriate credit to the original author(s) and the source, provide a link to the Creative Commons license, and indicate if changes were made. The images or other third party material in this article are included in the article's Creative Commons license, unless indicated otherwise in a credit line to the material. If material is not included in the article's Creative Commons license and your intended use is not permitted by statutory regulation or exceeds the permitted use, you will need to obtain permission directly from the copyright holder. To view a copy of this license, visit http://creativecommons. org/licenses/by/4.0/.

\section{References}

1. WHO. Obesity and overweight. World Heal. Organ. 2017. http://www.who.int/mediacentre/factsheets/fs311/en/.

2. Garvey WT, Mechanick JI, Brett EM, Garber AJ, Hurley DL, Jastreboff AM, et al. American association of clinical endocrinologists and American college of endocrinology comprehensive clinical practice guidelines for medical care of patients with obesity. Endocr Pract. 2016;22(Suppl 3):1-203.

3. Borgeraas H, Johnson LK, Skattebu J, Hertel JK, Hjelmesaeth J. Effects of probiotics on body weight, body mass index, fat mass and fat percentage in subjects with overweight or obesity: a systematic review and meta-analysis of randomized controlled trials. Obes Rev. 2018;19:219-32.

4. Zorzela L, Ardestani SK, McFarland LV, Vohra S. Is there a role for modified probiotics as beneficial microbes: a systematic review of the literature. Benef Microbes. 2017;8:739-54.

5. Martorell P, Llopis S, González N, Chenoll E, López-Carreras N, Aleixandre A, et al. Probiotic Strain Bifidobacterium animalis subsp. lactis CECT 8145 reduces fat content and modulates lipid metabolism and antioxidant response in Caenorhabditis elegans. $\mathbf{J}$ Agric Food Chem. 2016;64:3462-72.

6. Caimari A, del Bas JM, Boqué N, Crescentí A, Puiggròs F, Chenoll E, Martorell P, Ramón D, Genovés S, et al. Heat-killed Bifidobacterium animalis subsp. lactis CECT 8145 increases lean mass and ameliorates metabolic syndrome in cafeteria-fed obese rats. J Funct Foods. 2017;38:251-63.

7. Piepoli MF, Hoes AW, Agewall S, Albus C, Brotons C, Catapano AL, et al. 2016 European Guidelines on cardiovascular disease prevention in clinical practice. Eur J Prev Cardiol. 2016;23: NP1-NP96.

8. Vallbona CI, Roure CE, Violan FM AM. Guía de prescripció d'exercici físic per a la salut (PEFS). $2007 \mathrm{http}: / /$ canalsalut.gencat. cat/web/.content/home_canal_salut/professionals/temes_de_sa lut/activitat_fisica/documents/guiaprescripcioversiobreu.pdf.

9. Schaudinn A, Linder N, Garnov N, Kerlikowsky F, Blüher M, Dietrich A, et al. Predictive accuracy of single- and multi-slice MRI for the estimation of total visceral adipose tissue in overweight to severely obese patients. NMR Biomed. 2015; 28:583-90.

10. Motamed N, Perumal D, Zamani F, Ashrafi H, Haghjoo M, Saeedian FS, et al. Conicity index and waist-to-hip ratio are superior obesity indices in predicting 10-year cardiovascular risk among men and women. Clin Cardiol. 2015;38:527-34.

11. Codoñer FM, Ramírez-Bosca A, Climent E, Carrión-Gutierrez M, Guerrero M, Pérez-Orquín JM, et al. Gut microbial composition in patients with psoriasis. Sci Rep 2018; 8. https://doi.org/10.1038/ s41598-018-22125-y.

12. Kadooka Y, Sato M, Imaizumi K, Ogawa A, Ikuyama K, Akai Y, et al. Regulation of abdominal adiposity by probiotics (Lactobacillus gasseri SBT2055) in adults with obese tendencies in a randomized controlled trial. Eur J Clin Nutr. 2010;64:636-43.

13. Sharafedtinov KK, Plotnikova OA, Alexeeva RI, Sentsova TB, Songisepp E, Stsepetova J, et al. Hypocaloric diet supplemented with probiotic cheese improves body mass index and blood pressure indices of obese hypertensive patients-a randomized double-blind placebo-controlled pilot study. Nutr J. 2013;12:138.

14. Patnode CD, Evans CV, Senger CA, Redmond N, Lin JS. Behavioral counseling to promote a healthful diet and physical activity for cardiovascular disease prevention in adults without known cardiovascular disease risk factors: updated evidence report and systematic review for the US preventive services task force. JAMA. 2017;318:175-93.

15. Kadooka Y, Sato M, Ogawa A, Miyoshi M, Uenishi H, Ogawa H, et al. Effect of Lactobacillus gasseri SBT2055 in fermented milk on abdominal adiposity in adults in a randomised controlled trial. Br J Nutr. 2013;110:1696-703.

16. Sisti LG, Dajko M, Campanella P, Shkurti E, Ricciardi W, de Waure C. The effect of multifactorial lifestyle interventions on cardiovascular risk factors: a systematic review and meta-analysis of trials conducted in the general population and high risk groups. Prev Med (Baltim). 2018;109:82-97.

17. Gomes AC, de Sousa RGM, Botelho PB, Gomes TLN, Prada PO, Mota JF. The additional effects of a probiotic mix on abdominal adiposity and antioxidant status: a double-blind, randomized trial. Obesity. 2017;25:30-8.

18. Takahashi S, Anzawa D, Takami K, Ishizuka A, Mawatari T, Kamikado K, et al. Effect of Bifidobacterium animalis ssp. lactis GCL2505 on visceral fat accumulation in healthy Japanese adults: a randomized controlled trial. Biosci Micro, Food Heal. 2016;35:163-71.

19. Chen M-F, Weng K-F, Huang S-Y, Liu Y-C, Tseng S-N, Ojcius $\mathrm{DM}$, et al. Pretreatment with a heat-killed probiotic modulates monocyte chemoattractant protein- 1 and reduces the pathogenicity of influenza and enterovirus 71 infections. Mucosal Immunol. 2017;10:215-27.

20. Bagarolli RA, Tobar N, Oliveira AG, Araújo TG, Carvalho BM, Rocha GZ, et al. Probiotics modulate gut microbiota and improve insulin sensitivity in DIO mice. J Nutr Biochem. 2017;50:16-25. 
21. Van Harmelen V, Reynisdottir S, Eriksson P, Thörne A, Hoffstedt J, Lönnqvist $\mathrm{F}$, et al. Leptin secretion from subcutaneous and visceral adipose tissue in women. Diabetes. 1998;47:913-7.

22. Everard A, Belzer C, Geurts L, Ouwerkerk JP, Druart C, Bindels LB, et al. Cross-talk between Akkermansia muciniphila and intestinal epithelium controls diet-induced obesity. Proc Natl Acad Sci. 2013;110:9066-71.

23. Cani PD, Amar J, Iglesias MA, Poggi M, Knauf C, Bastelica D, et al. Metabolic endotoxemia initiates obesity and insulin resistance. Diabetes. 2007;56:1761-72.

\section{Affiliations}

\section{Anna Pedret ${ }^{1,2} \cdot$ Rosa M. Valls ${ }^{2,1} \cdot$ Lorena Calderón-Pérez $^{2,1} \cdot$ Elisabet Llauradó $^{2,1} \cdot$ Judit Companys $^{1,2}$. Laura Pla-Pagà $^{1,2}$ - Ana Moragas ${ }^{4,5,6} \cdot$ Francisco Martín-Luján $^{4,5,7} \cdot$ Yolanda Ortega $^{4,5,8} \cdot$ Montse Giralt $^{2,1}$.

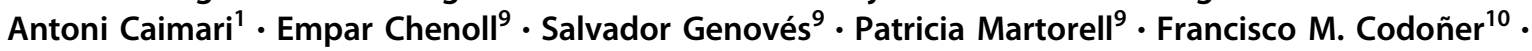 Daniel Ramón ${ }^{9,1} \cdot$ Lluís Arola $^{1,11} \cdot$ Rosa Solà ${ }^{1,2,3}$}

1 Eurecat, Centre Tecnològic de Catalunya, Unitat de Nutrició i Salut, Reus, Spain

2 Universitat Rovira i Virgili, Facultat de Medicina i Ciències de la Salut, Functional Nutrition, Oxidation, and Cardiovascular Diseases Group (NFOC-Salut), Reus, Spain

3 Hospital Universitari Sant Joan de Reus, Reus, Spain

4 Departament de Medicina i Cirurgia, Universitat Rovira i Virgili, Reus, Spain

5 Institut Universitari d'Investigació en Atenció Primària-IDIAP Jordi Gol, Tarragona, Spain

6 Primary Care Centre Jaume I, Institut Català de la Salut, Tarragona, Spain
7 Primary Care Centre El Morell, Institut Català de la Salut, Tarragona, Spain

8 Primary Care Centre Salou, Institut Català de la Salut, Tarragona, Spain

9 Biopolis SL/Archer Daniels Midland. R\&D Department (ADM Nutrition). C/Catedrático Agustín Escardino Benlloch 9, 46980Paterna Valencia, Spain

10 Lifesequencing/Archer Daniels Midland. R\&D Department (ADM Nutrition). C/Catedrático Agustín Escardino Benlloch 9, 46980Paterna Valencia, Spain

11 Universitat Rovira i Virgili, Facultat de Química, Grup de Recerca en Nutrigenòmica, Tarragona, Spain 